\author{
Paper for the Special Session on Nanostructured Materials at the \\ $4^{\text {th }}$ AIAA/ASME/ASCE/AHS/ASC
}

Structures, Structural Dynamics and Materials Conference and Exhibit

\title{
A Statistical Approach for the Concurrent Coupling of Molecular Dynamics and Finite Element Methods
}

\author{
E. Saether ${ }^{1}$, V. Yamakov ${ }^{2}$, and E. Glaessgen ${ }^{3}$ \\ NASA Langley Research Center, Hampton, VA, 23681
}

\begin{abstract}
Molecular dynamics (MD) methods are opening new opportunities for simulating the fundamental processes of material behavior at the atomistic level. However, increasing the size of the MD domain quickly presents intractable computational demands. A robust approach to surmount this computational limitation has been to unite continuum modeling procedures such as the finite element method (FEM) with MD analyses thereby reducing the region of atomic scale refinement. The challenging problem is to seamlessly connect the two inherently different simulation techniques at their interface. In the present work, a new approach to MD-FEM coupling is developed based on a restatement of the typical boundary value problem used to define a coupled domain. The method uses statistical averaging of the atomistic MD domain to provide displacement interface boundary conditions to the surrounding continuum FEM region, which, in return, generates interface reaction forces applied as piecewise constant traction boundary conditions to the MD domain. The two systems are computationally disconnected and communicate only through a continuous update of their boundary conditions. With the use of statistical averages of the atomistic quantities to couple the two computational schemes, the developed approach is referred to as an embedded statistical coupling method (ESCM) as opposed to a direct coupling method where interface atoms and FEM nodes are individually related. The methodology is inherently applicable to three-dimensional domains, avoids discretization of the continuum model down to atomic scales, and permits arbitrary temperatures to be applied.
\end{abstract}

\section{Introduction}

The emerging field of nanomechanics is providing a new focus in the study of the mechanics of materials: That of simulating fundamental atomic mechanisms involved in the initiation and evolution of damage. These simulations are commonly based on either quantum mechanics (ab-initio, tight-binding (TB), density-functional theory (DFT)) methods or on classical molecular dynamics (MD) and molecular statics (MS) methods. Predictions of material behavior at nanometer length scales promise the development of physics-based 'bottom-up' multiscale analyses that can aid in understanding the evolution of failure mechanisms across length scales. However, modeling atomistic processes quickly becomes computationally intractable as the system size increases. With current computer technology, the computational demands of modeling suitable domain sizes (on the order of hundreds of atoms for quantum mechanics based methods, and potentially billions of atoms for classical mechanics based methods) and integrating the governing equations of state over sufficiently long time intervals quickly reaches an upper bound for practical analyses. In contrast, continuum mechanics methods such as the finite element method (FEM) provide an economical numerical representation of material behavior at length scales in which continuum assumptions apply.

\footnotetext{
${ }^{1}$ Durability, Damage Tolerance, and Reliability Branch, MS/188E, AIAA Member.

${ }^{2}$ National Institute for Aerospace.

${ }^{3}$ Durability, Damage Tolerance, and Reliability Branch, MS/188E, AIAA Associate Fellow.
} 
The concept of bridging length scales by coupling different computational paradigms is particularly attractive as a highly efficient means of reducing the computational cost of the simulations in cases that require modeling of relatively large material domains to capture the complete deformation field, but where atomic and subatomic refinement is needed only in very localized regions (e.g. near a crack tip or dislocation core). Such computational issues arise in modeling crack nucleation and propagation, and in modeling dislocation formation and interaction. By using coupled models, the size limitations of the atomistic simulation can be avoided by embedding an inner atomistic domain where complex dynamic processes and large deformation gradients exist within an outer domain where the deformation gradients are small so that a continuum representation of the material becomes appropriate.

Over the past decade, various methods that couple material representations at different levels of refinement have been developed and offer significant computational advantages compared with full MD simulations for predicting deformation and fracture processes ${ }^{1-11}$. In all of these methods, the most challenging problem is the computational connection of the two different material representations at an imaginary interface where the continuity of the material properties and kinematics must be preserved during simulation. For example, the coupling between the subatomic quantum mechanics description and the classical atomistic mechanics representation requires a transition from a highly interdependent representation of the state of the whole system, which cannot be partitioned into individual atomic states, to the classical representation of individual atoms interacting through Newtonian forces. A way to resolve this challenge was provided by the work by Abraham and co-workers ${ }^{1,2}$ who developed a procedure that combines semi-empirical TB calculations, that account for the electronic structure as parameterized from firstprinciples of quantum mechanics, with a MD simulation based on Newtonian mechanics. The procedure was used to simulate the intricate mechanisms of atomic debonding at the tip of a propagating crack in silicon.

No less challenging is the coupling between atomistic and continuum material representations. In this case, continuity of material properties must be maintained while transitioning from individual atoms interacting through nonlocal forces to the local stress-strain field formalism of continuum mechanics. Because of the potentially enormous computational benefit that might be achieved by replacing portions of the discrete atomistic domain with a continuum representation, allowing for orders of magnitude increase in the system size without a substantial increase of the computational cost, the atomistic - continuum coupling is attracting increased attention. Various specialized coupling methods ${ }^{3-5}$ have been developed to address different problems (e.g. crack propagation, dislocation evolution) in a computationally efficient manner.

In one formulation, named the coupling of length scales method (CLS $)^{2}$, the nodes in a finite element model (FEM) representing the continuum region are directly connected to the atoms of an atomistic region forming an interface of "pad" atoms. In another formulation, called the quasicontinuum (QC) method, reviewed by Miller and Tadmor ${ }^{6}$, the regions to be represented as either atomistic or continuum are determined by evaluating the magnitude of evolving local deformations. The representations of different regions are performed using representative atoms or "repatoms". In the QC formalism, "nonlocal repatoms" are used to represent "real" atoms to form atomistic regions treated by MS/MD methods while "local repatoms" are used to define continuum domains using FEM procedures. Another representative coupling approach is the bridging method of Xiao and Belytschko ${ }^{7}$ which is based on an overlay approach in which MD and FEM representations are superposed. The coupled atomistic/dislocation dynamics (CADD) method of Shilkrot et al. ${ }^{8}$ is specifically designed to simulate, identify and pass dislocations between atomistic and continuum domains. For crack problems, the early efforts of Gumbsch and Beltz led to the development of the finite element-atomistic (FEAt) coupling procedure that combined an embedded MD region with a finite element domain. A generalized formulation of the conventional FEM, which allows FEM nodes to be considered as coarse-grained MD "atoms", led to another computational scheme for atomistic-continuum coupling called coarse grained molecular dynamics (CGMD). A detailed representation of CGMD is given by Rudd and Broughton $^{10,11}$. Other variations and modifications of the above referenced approaches also exist.

A common feature of all these approaches ${ }^{1-11}$ for coupling atomistic with continuum representation is the refinement of the FEM mesh down to atomic scale and make a transition from FEM mesh nodes to discrete atoms at the interface. The approaches of relating the kinematics of atoms and FEM nodes in a one-to-one manner will be referred to in this paper as direct coupling (DC) approaches.

While DC approaches are straightforward, the fundamental difficulty in their development lies in the inherent differences between the atomistic and continuum computational models. The physical state of the atomistic region is described through nonlocal interatomic forces between discrete atoms of given position and momentum, while the 
physical state of the continuum region is described through continuous stress-strain fields that reflect local statistical averages of atomic interactions at larger length and time scales. Using a continuum representation at atomic length scales creates potential difficulties, such as the appearance of "ghost" forces ${ }^{3-5}$ or the imposition of nonphysical constraints on the motion of atoms at the interface that are equated to the displacements of the continuum mesh rather than to the dynamical effects of interatomic forces. In general, the formal connection between continuum and discrete quantities can only be achieved through an adequate statistical averaging over scales where the discreteness of the atomic structure can be successfully approximated as a continuum.

In this paper, an alternative approach to the DC approaches is proposed to construct a coupled MDFEM system. The approach is based on solving the special boundary value problem (BVP) at the MD-FEM interface for a MD system embedded in a FEM domain as depicted in Figure 1.

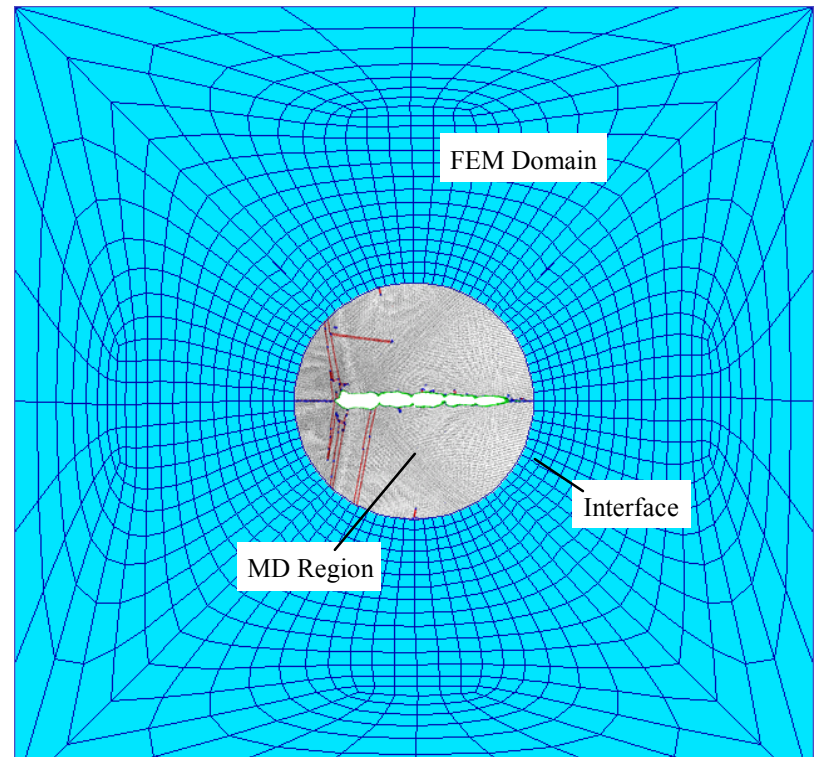

Figure 1. An embedded MD region within an FEM domain.

The method uses statistical averaging over both time and volume of atomistic subdomains at the MD-FEM interface to provide nodal displacement boundary conditions to the continuum FEM domain, which, in turn, generates interface reaction forces that are applied as constant traction boundary conditions ${ }^{12}$ to the atoms within the localized MD subdomain. Thus, this approach may be described as a local-nonlocal BVP because it relates local continuum nodal quantities with nonlocal statistical averages of atomistic quantities over selected atomic subdomains. An iterative procedure between the MD statistical displacements and the FEM reaction forces ensures continuity at the interface. In this way, the problem of redefining continuum variables at the atomic scale is avoided, and the developed interface approach links different time and length scales between the MD and FEM domains. Typically, one finite element at the interface encompasses a region of several hundred to several thousand atoms. At this scale, the discreteness of the atomic structure is homogenized enough so that the FEM domain responds to the atomistic domain as an extension of the continuum. At the same time, the piecewise constant traction boundary conditions of the MD domain ensure that the elastic field from the FEM domain is correctly transferred to the atomistic region. With the use of statistical averages to couple the two computational schemes, the developed approach is referred to as a statistical coupling (SC) approach as opposed to the DC approaches. Based on the SC approach, the developed MD-FEM coupling method is referred to as the embedded statistical coupling method (ESCM).

This paper will detail the ESCM approach to coupling MD and FEM computational domains for the case of systems that reach thermodynamic equilibrium or evolve quasistatically. While there is no principle difficulty in implementing this approach for non-equilibrium systems, it is beneficial to consider the case of equilibrium simulations first to illustrate the methodology.

Section II.A describes the structure of the coupled MD-FEM model. Sections II.B and II.C discuss elements of the coupling interface involving the Interface MD Region and Surface MD Region, respectively. Section II.D presents an overview of the overall iterative MD-FEM coupling methodology. Section III presents several validation studies to substantiate the accuracy of the developed coupling methodology. Finally, Section IV will present concluding remarks on the overall effectiveness of the ESCM.

\section{The ESCM Model}

The ESCM model consists of four regions: the inner MD region, the interface MD region, the surface MD region and the FEM mesh as shown in Figure 2. The inner MD region consists entirely of an atomistic representation of the material. The interface MD/FE region consists of the interface MD region and the interface FEM nodes and 


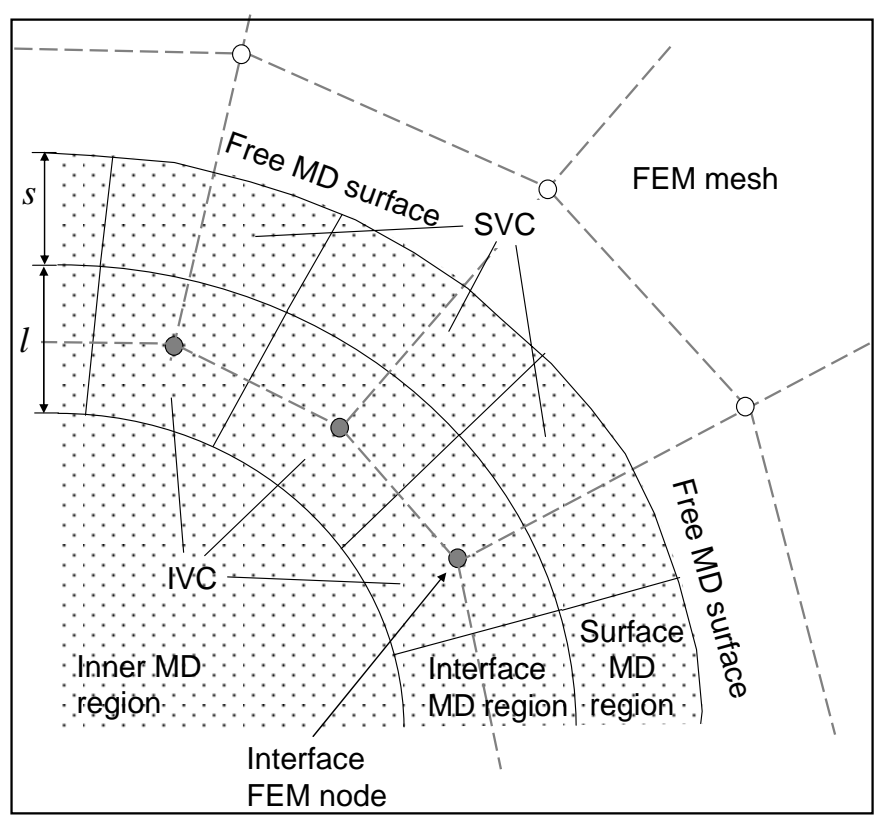

Figure 2. Structure of the ESCM. performs the primary coupling of MD and FEM domains via the ESCM statistical interfacing procedures. The surface MD region extends to the outer boundaries of the atomistic simulation and acts to approximate the internal forces of an infinite MD domain. Together, the inner MD region, the interface $\mathrm{MD}$ region and the surface MD region comprise the MD domain. Likewise, the FEM domain consists entirely of a continuum representation of the material and extends from the interface FEM nodes to the outer boundaries of the simulation.

\section{A. MD and FEM model components}

The inner MD region is used to model material phenomena at the atomistic level and is simulated under the full influence of the FEM reaction forces applied as traction boundary conditions to the interface region. This inner MD region should be large enough to sustain any of the types of processes (mostly plastic deformation events such as dislocation or void nucleation, crack propagation, etc.) that are required by the simulation.

It is important to emphasize that the partitioning of the MD system into an inner region, an interface region and a surface region is not a physical separation of the system. An atom assigned to a particular location freely interacts with atoms in its interaction neighborhood that may reside in a different region. Thus, the overall simulation is performed using any conventional MD technique and the whole MD configuration is treated as an independent integrated MD system without any imposition of direct kinematic constraints. The only difference between the three MD regions is that, while the atoms in the inner MD region are subject only to their interatomic forces, the interface and surface MD regions are used to facilitate the application of external forces involved in ESCM coupling that act in addition to the interatomic forces. The modification of the MD computations relates only to the application of external forces and will be discussed in Sections II.B and II.C where details of the interface and surface MD regions are presented.

The FEM domain is used to replace an atomistic representation with a continuum model in parts of the domain where the deformation gradients are small and atomic level resolution is not necessary. Using a FEM model permits a large reduction in the computational cost of performing MD simulations by limiting the atomic resolution to nonlinear processes in the inner MD region. The current application uses the FEM domain to simulate an extended material model such that the elastic deformation and load transfer due to applied far-field boundary conditions are accurately transferred to the inner MD region. The continuum field is currently assumed to be static with linear elastic material properties but other applications of ESCM might require the incorporation of nonlinear material behavior such as plasticity or general dynamic response such that nonlinear processes generated in the inner MD domain could be propagated into the continuum.

\section{B. Interface MD region}

The main role of the interface MD region (Figure 2) is to provide the connection between the MD and the FEM domains. The interface MD region is formed by atoms that surround those FEM nodes, called interface FEM nodes, that overlap the MD domain. The atoms that surround a given interface node form a cell in the interface MD region, called an interface volume cell (IVC). The IVCs are established to communicate averaged MD displacements computed at their mass center to the FEM nodes, and to provide a set of atoms over which FEM reaction forces are distributed to the MD system during simulation. The IVCs need not coincide in size and shape with the finite element to which the FEM node belongs. In the model described in this paper, the IVCs are formed through a Voronoi-type construction ${ }^{13}$ by selecting those atoms with a common closest FEM node. Accordingly, the average 
size of the IVCs becomes equal to the average distance between the interface FEM nodes, $l$, which also defines the in-plane thickness of the interface MD region (Figure 2).

During subsequent coupled MD-FEM simulation, the volume of the IVC is the integration volume for the statistical displacement vector $\vec{\delta}_{I}$, that is prescribed to the interface FEM node. The displacement vector for each interface FEM node is calculated as a time-average of the center of mass displacement $\delta_{\mathrm{CM}}$ of the IVC over a certain period of $M$ MD steps

$$
\vec{\delta}_{I}=\left\langle\vec{\delta}_{C M}\right\rangle_{t}=\frac{1}{M} \sum_{m=1}^{M}\left(\vec{r}_{C M}\left(t_{m}\right)-\vec{r}_{C M}(0)\right)
$$

In the above expression, $\vec{r}_{C M}\left(t_{m}\right)=\frac{1}{N} \sum_{i=1}^{N} \vec{r}^{(i)}\left(t_{m}\right)$ is the center of mass of the IVC containing $N$ atoms at positions $\vec{r}^{(i)}$ at time $t_{\mathrm{m}}$ of the $m^{\text {th }}$ MD step.

In Eq. (1), the mass center displacement $\vec{\delta}_{C M}$ is calculated relative to the initial zero-displacement position of the IVC. This initial position is defined as the location of the mass center of the IVC when the MD system is equilibrated at zero applied load.

The equilibration at zero applied load cannot be done using a MD domain with a free surface because the surface tension would produce a pressure, $p_{\mathrm{s}}$, on the surface resulting in erroneous zero-displacement positions for the IVC mass centers. To avoid surface tension effects, the zero displacements are estimated by using a MD system of a rectangular shape that is equilibrated under periodic boundary conditions in all three dimensions at zero pressure. The details of the equilibration procedure will be given in a separate publication ${ }^{14}$.

Both the reaction forces calculated at the interface FEM nodes in response to the provided displacements and the MD interatomic forces are applied uniformly on the atoms of the IVCs as external forces. The application of the FEM reaction forces to the IVCs can generate undesired phonons or resonant elastic oscillations in the dynamic MD domain. These oscillations must be damped in order to achieve equilibrium with the static FEM region. A number of different damping schemes have been addressed in the literature. Holian and Ravelo ${ }^{15}$, and more recently, Schäfer et al. ${ }^{16}$, found that applying viscous damping to the atoms in a region surrounding the center of the MD system can effectively absorb the intense phonon waves coming from a propagating crack. In this scheme, a friction force, $\vec{\eta}$, is given by

$$
\vec{\eta}=-\chi \overrightarrow{\mathbf{v}}
$$

and is applied to the atoms of the damped region in proportion to the atom's velocity $\vec{v}$ and an appropriately chosen viscous coefficient $\chi^{15,16}$. The method is efficient and simple to implement. Its drawback is that it effectively decreases the local temperature in the damping region resulting in undesirable strain gradients because of the thermal expansion.

To avoid thermal effects, the damping used in the present method is based on a modified form of Equation (2), where instead of being proportional to each individual atom's velocity, $\vec{\eta}$ is set proportional to the group velocity of the mass center of a certain volume of $N$ atoms. The frictional force, $\vec{\eta}$, and the group velocity of the mass center, $\vec{v}_{c m}$, are given by

$$
\vec{\eta}=-\chi \overrightarrow{\mathbf{v}}_{c m} ; \quad \overrightarrow{\mathbf{v}}_{c m}=\frac{1}{N} \sum_{i=1}^{N} \overrightarrow{\mathbf{v}}_{i}
$$


By controlling the size of this volume, one can damp phonons of wavelengths larger than the volume size while leaving the shorter wavelengths associated with random thermal fluctuations unaffected as their contribution to the group velocity averages to zero. Phonons introduced by the FEM mesh cannot have wavelengths smaller than the distance $l$ between the interface FEM nodes (Figure 2). Thus, it is convenient to choose the IVCs at the interface MD region and corresponding surface volume cells (SVCs, see Figure 2) at the surface MD region (to be discussed in Section II.C) as the volumes in which damping is applied. For the models used in the present work (to be discussed in Section III), effective viscous wave damping was achieved by setting $\chi=3 \mathrm{eVps} / \mathrm{nm}^{2}$. For this $\chi$, and for an IVC and SVC thickness of $l$ and s, respectively, on the order of $3 \mathrm{~nm}$, the effective temperature in the damping volumes decreased only by $1 \%$ compared to the bulk temperature. A discussion of criteria for the selection of the value of the viscous coefficient, $\chi$, can be found in Reference 16. In those instances where viscous wave damping is not adequate (e.g. collisional dynamics), the more precise non-reflective boundary condition techniques discussed in References 17-19 can be implemented.

\section{Surface MD region}

The proper embedding of a localized MD domain within a coupled FEM domain should generate a force environment within the inner MD region that closely approximates the internal forces of an infinite MD domain subjected to externally applied far-field loading. In ESCM, external forces are transferred from the surrounding FEM domain into the MD region as nodal reaction forces distributed to the atoms of the IVCs in the MD interface region. In order for the MD domain to deform freely in response to these reaction forces, it is simulated under free surface boundary conditions. The existence of a free surface introduces several undesirable effects in the MD system. First, it creates surface tension forces that must be removed to avoid distorting the MD response. Second, because atoms that should lie within the cutoff radius of the surface atom's potential are missing, the surface atoms are less strongly bonded to the material than the bulk atoms. Under sufficiently high reaction forces, these atoms may be separated from the surface layer causing a surface degradation of the MD domain. To mitigate these free surface effects, a surface MD region - as shown in Figure 3 - is introduced. This additional region serves several necessary functions. First, positioning the outer free surface away from the interface MD region stabilizes the atomic structure of the interface MD region against strong FEM reaction forces produced during potentially large deformations. Second, the surface MD region is conveniently used as a volume where an additional external force is applied to counteract the surface tension force and to eliminate the surface tension effects on the remainder of the MD domain.

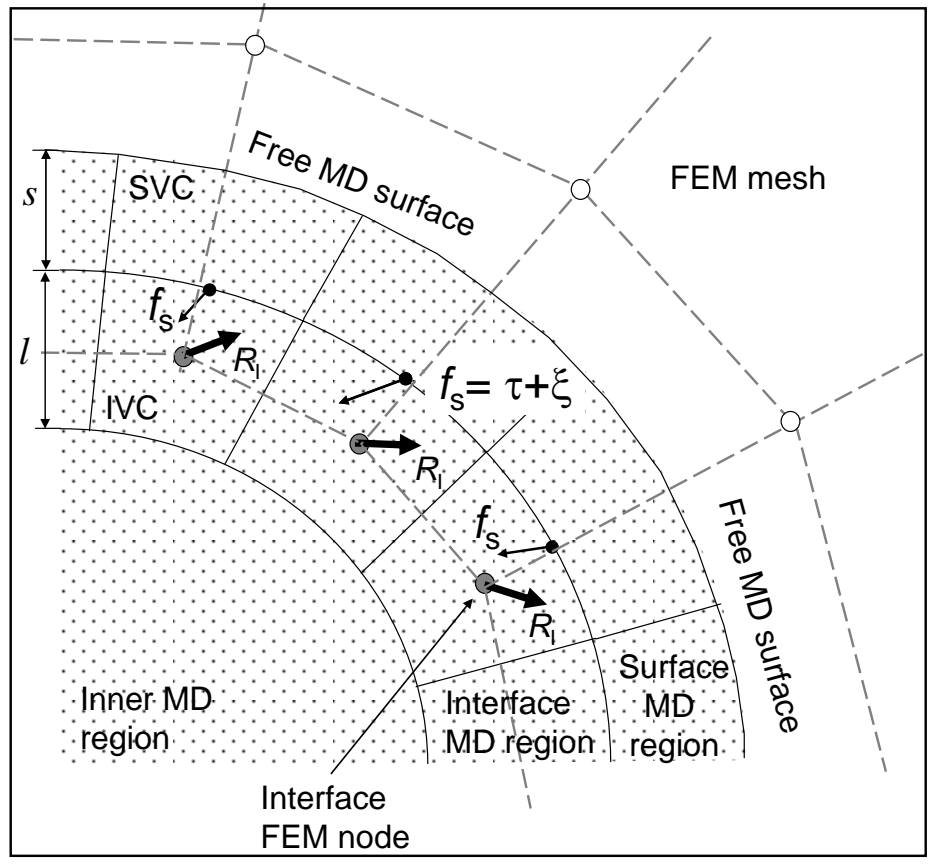

Figure 3. Generation of surface shell forces.

However, this surface region introduces an undesirable fictitious stiffness outside the perimeter of the applied FEM nodal forces which elastically constrains the deformation of the inner MD domain. The combined effect of the surface tension and the stiffness of the surface MD region may be defined as a resultant force, $f_{\mathrm{s}}$, which acts at the boundary with the interface MD region and is given by the sum of two components expressed as

$$
\vec{f}_{s}=\vec{\xi}+\vec{\tau}
$$

In this equation, $\vec{\xi}$ is the elastic reaction of the surface MD region under deformation, and $\vec{\tau}$ is the force that results from the surface tension (Figure 3). 
A way to mitigate both the surface tension and the elastic response of the surface MD region is to estimate and compensate the force $f_{\mathrm{s}}$. In the ideal case, when $f_{\mathrm{s}}$ is fully compensated, the surface MD region acts as if it possesses zero stiffness and no surface tension, thereby mitigating spurious influences on the MD system. To follow the variations of $f_{\mathrm{s}}$ along the perimeter of the MD domain, the surface region is divided into a number of SVCs. The $f_{\mathrm{s}}$ is calculated individually for each SVC. The force compensation is performed by calculating a counterforce, $f_{\mathrm{c}}$, along the IVC/SVC interface and applying this force over the atoms of each SVC in a similar way as the reaction nodal forces are applied to the IVCs of the interface MD region. Although the SVCs do not have to correlate in position and size with the IVCs, it may be computationally convenient to form SVCs as extensions of the IVCs into the surface region. The details of the force compensation method used are fully described in a separate publication ${ }^{14}$.

\section{MD-FEM coupling}

The MD-FEM coupling in the ESCM is achieved through an iterative equilibration scheme between the MD domain and the FEM region. In this scheme, the MD domain is simulated under constant traction boundary conditions, provided by the FEM as interface reaction forces $R_{I}$. After a selected period of MD simulation, during which the MD domain responds dynamically to $R_{I}$, the displacements at the MD-FEM interface are calculated as statistical averages over the atomic positions and are averaged over time.

These average displacements are imposed as displacement boundary conditions $\delta_{I}$ for the FEM region. The FEM problem is then solved to recover new interface reaction forces $R_{I}$, resulting from the interface displacements $\delta_{I}$ and the imposed far-field displacement boundary conditions $\delta_{F}$, which define the total loading conditions of the coupled system. The new interface reaction forces $R_{I}$ are distributed to the interface atoms in the IVCs of the interface MD zone, defining new constant traction boundary conditions. The next period of MD simulation then takes place. Updated interface displacements are calculated and, again, provided as displacement boundary conditions to the FEM. This MD-FEM iteration cycle repeats during which a stable equilibrium of both displacements and forces between the atomistic and continuum material fields at the interface is established. The period of MD simulation prior to FEM update of $R_{I}$ is selected by a determination of the convergence rate to a stable equilibrium state.

The basic equations of the ESCM coupling methodology are now developed. The elastic properties of the material in the FEM region are described through a set of stiffness matrices $\left[K_{\alpha \beta}\right]$, with $\alpha, \beta=V, F, I$ indicating: $V-$ variables within the interior of the FEM region; $F$ - far-field variables; and $I$ - interface variables. Using these definitions, the static continuum equations of state at the $n^{\text {th }}$ FEM update are given by

$$
\left[\begin{array}{lll}
{\left[K_{V V}\right]} & {\left[K_{V F}\right]} & {\left[K_{V I}\right]} \\
{\left[K_{F V}\right]} & {\left[K_{F F}\right]} & {\left[K_{F I}\right]} \\
{\left[K_{I V}\right]} & {\left[K_{I F}\right]} & {\left[K_{I I}\right]}
\end{array}\right]\left\{\begin{array}{l}
\left\{\delta_{V}\left(t_{n}\right)\right\} \\
\left\{\delta_{F}\left(t_{n}\right)\right\} \\
\left\{\delta_{I}\left(t_{n}\right)\right\}
\end{array}\right\}=\left\{\begin{array}{l}
\left\{R_{V}\left(t_{n}\right)\right\} \\
\left\{R_{F}\left(t_{n}\right)\right\} \\
\left\{R_{I}\left(t_{n}\right)\right\}
\end{array}\right\}
$$

To ensure that the FEM region has the same elastic properties as the MD domain, the stiffness terms in the $\left[K_{\alpha \beta}\right]$ submatrices are calculated from the anisotropic elastic constants derived from the MD interatomic potential at the temperature of the simulation ${ }^{20}$. The FEM model is subjected to two types of displacement boundary conditions: (i) the far-field displacements $\left\{\delta_{F}\right\}$, which define the load over the entire coupled MD-FEM system; and (ii) the interface displacements $\left\{\delta_{I}\right\}=\left(\delta_{I}^{1}, \delta_{I}^{2}, . . \delta_{I}^{k}, ..\right)$, which represent the deformation response of the MD domain at the $1^{\text {st }}, 2^{\text {nd }}, \ldots$, and $k^{\text {th }} \mathrm{IVC}$.

The solution for the unknown displacements in the interior of the FEM domain, $\left\{\delta_{V}\right\}$, is given by

$$
\left\{\delta_{V}\left(t_{n}\right)\right\}=\left[K_{V V}\right]^{-1}\left(\left\{R_{v}\right\}-\left[K_{V F}\right]\left\{\delta_{F}\left(t_{n}\right)\right\}+\left[K_{V I}\right]\left\{\delta_{I}\left(t_{n}\right)\right\}\right),
$$


which allows the calculation of the interface reaction forces, $\left\{R_{I}\left(t_{n}\right)\right\}=\left(R_{I}^{1}, R_{I}^{2}, . . R_{I}^{k}, ..\right)$ of the $1^{\text {st }}, 2^{\text {nd }}, \ldots$, and $k^{\text {th }}$ IVC to be obtained from

$$
\left\{R_{I}\left(t_{n}\right)\right\}=\left[K_{I V}\right]\left\{\delta_{V}\left(t_{n}\right)\right\}+\left[K_{I F}\right] \quad\left\{\delta_{F}\left(t_{n}\right)\right\}+\left[K_{I I}\right]\left\{\delta_{I}\left(t_{n}\right)\right\},
$$

together with the far-field forces of constraint

$$
\left\{R_{F}\left(t_{n}\right)\right\}=\left[K_{F V}\right]\left\{\delta_{V}\left(t_{n}\right)\right\}+\left[K_{F F}\right] \quad\left\{\delta_{F}\left(t_{n}\right)\right\}+\left[K_{F I}\right]\left\{\delta_{I}\left(t_{n}\right)\right\} .
$$

The dynamics of an atom $i$ of mass $\mu^{(i)}$ at position $r^{(i)}$ in the embedded MD domain is described by Newton's equations of motion

$$
\mid \begin{aligned}
& \mu^{(i)} \ddot{r}^{(i)}=f^{(i)} ; \quad i \in(\text { inner } \quad \text { MD region }) \\
& \mu^{(i)} \ddot{r}^{(i)}=f^{(i)}+R_{I}^{k} / N_{I}^{k}-\chi \mathbf{v}_{c m}^{k} ; \quad i \in(I V C)_{k} \quad \text { interface shell } \\
& \mu^{(i)} \ddot{r}^{(i)}=f^{(i)}+f_{c}^{k} / N_{S}^{k}-\chi \mathbf{v}_{c m}^{k} ; \quad i \in(\mathrm{SVC})_{k} \quad \text { surface shell }
\end{aligned}
$$

where $\chi$ and $v_{c m}$ are defined in Equation (3).

Convergence of the interface forces $\left\{R_{I}\right\}$ and displacements $\left\{\delta_{I}\right\}$ can be achieved only if the MD system can reach a force balance with the exterior FEM system subject to the far-field displacement constraints. At equilibrium, first, $\mathbf{v}_{c m}^{k}=0$, and second, the change of the total momentum $\Delta p_{\mathrm{k}}$ of any $k^{\text {th }}$ IVC due to the FEM reaction force for the period of the MD run of $M$ time steps is also zero

$$
\Delta p_{k}=\sum_{n=1}^{M} \sum_{i=1}^{N_{I}^{k}} \mu^{(i)} \ddot{r}^{(i)}\left(t_{n}\right) \Delta t=0
$$

Performing the same double summation to the second equation in (8) results in

$$
\frac{1}{M} \sum_{m=1}^{M} \sum_{i=1}^{N_{I}^{k}} f^{(i)}\left(t_{m}\right)=\left\langle\sum_{i=1}^{N_{I}^{k}} f^{(i)}\right\rangle_{t}=-R_{I}^{k}
$$

which means that, at equilibrium, the FEM reaction force becomes equal and opposite to the average MD atomic force in the corresponding $k^{\text {th }}$ IVC. Equation (10) presents the establishment of static force equilibrium between the MD domain and the FEM region.

\section{Test Simulations of the ESCM}

\section{A. The simulation models}

A series of tests is presented in this section to examine the performance of the ESCM. The model geometry used in all the tests is shown in Figure 4. This model consists of an inner circular MD domain of diameter $d_{\mathrm{MD}}$ which is embedded into a larger exterior square FEM domain of elastic material with a side dimension of $d_{\mathrm{FE}}=$ $20 d_{\mathrm{MD}}$. The specifics of the MD and the FEM models and simulations will be discussed next, followed by the results and analyses of the test simulations. 


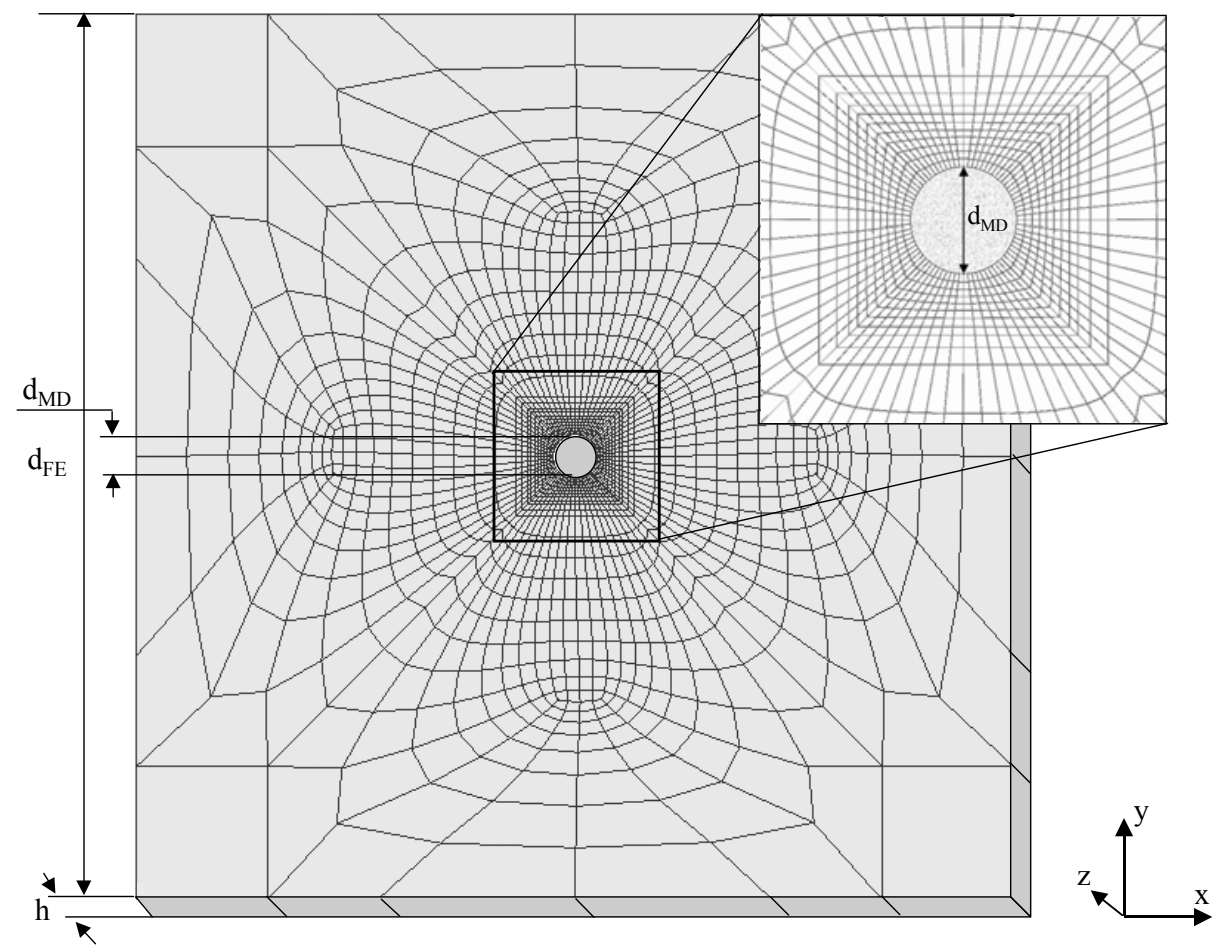

Figure 4. Coupled MD-FEM model.

\section{The MD model}

The material for the simulation models is chosen to be a perfect crystal of aluminum. The atomic properties of aluminum are represented by the embedded atom model (EAM) potential of Mishin et al. ${ }^{21}$, which is fitted to give the correct zero-temperature lattice constant, $a_{\mathrm{o}}=0.405 \mathrm{~nm}$, elastic constants, cohesive energy, vacancy formation energy, etc. A 2000 point tabulation of the potential function in the interval of interatomic distances from $r_{\min }=$ $0.25 a_{\mathrm{o}}(0.1 \mathrm{~nm})$ to the cut-off radius, $r_{\mathrm{c}}=1.55 a_{\mathrm{o}}(0.628 \mathrm{~nm})$ was used.

In this study, a second order polynomial interpolation for the tabulated EAM potential was applied, which, combined with a high precision fifth order Gear predictor-corrector integration scheme for calculating the atomic trajectories ${ }^{22}$, gave errors in estimating the stress in the system under uniaxial strain in the range of $0.1-0.5 \%$ that were less than $0.5 \mathrm{MPa}$. This is equivalent to detecting a change in strain of the order of $0.007 \%$. The high accuracy in strain detection allowed accurate measurements of the MD-FEM coupled model at relatively small strains of 0.1 to $0.5 \%$.

The first test simulation used a MD system in the form of a circular plate of monocrystalline aluminum with its main crystallographic axes [ $\left[\begin{array}{lll}1 & 0 & 0\end{array}\right],\left[\begin{array}{lll}0 & 1 & 0\end{array}\right]$ and $\left[\begin{array}{lll}0 & 0 & 1\end{array}\right]$ oriented along the $x$-, $y$ - and $z$ - directions, respectively (Figure 4). The system was simulated under periodic boundary conditions along the $z$ - direction and free surface boundary conditions along its perimeter. These boundary conditions allow the MD domain to deform in an unconstrained manner in the $x-y$ plane under the external reaction forces from the FEM region while maintaining constant zero pressure along the $z$-direction using the Parrinello-Rahman constant-pressure simulation technique ${ }^{23}$. Constant temperature is maintained by applying the Nose-Hoover thermostat ${ }^{24}$. The tests were performed at near zero temperature $(T=10 \mathrm{~K})$ to minimize the thermal noise, and at room temperature $(T=300 \mathrm{~K})$ to demonstrate the ESCM for more practically relevant situations in which thermal effects are important.

The thickness of the plate along $z$ is equal to the thickness, $h=5 a_{0} \approx 2.0 \mathrm{~nm}$, of the coupled MD-FEM system as shown in Figure 4. Though very thin, the MD domain mechanically behaves as an infinitely thick plate due to the applied periodic boundary conditions along the $z$ - direction. The interference of the atoms with their periodic 
images in the $z$-direction is prevented by the fact that $h$ is more than three times larger than the range of the interatomic potential $r_{\mathrm{c}}$ thus preserving the local three-dimensional characteristics of the system.

Although the ESCM approach can be applied to an arbitrary interface geometry, the circular shape of the MD domain was chosen for several reasons. First, the circular shape is the equilibrium shape of a free body with respect to the surface tension. As already discussed in Section II, in the ESCM approach the MD system represents a free body placed in the elastic force field generated by the continuum FEM domain. The MD-FEM coupling is more effective if the free body is in equilibrium with itself before the coupling is imposed, which is not guaranteed for an MD domain of general shape. Starting with a nonequilibrium shape of the MD region may cause the applied counter-forces to be significant compared to the interatomic forces and, thus, may significantly affect the atomic state. Second, the MD-FEM interface is uniform everywhere around the MD system, which significantly simplifies the analyses of its behavior and the surface tension compensation procedure as described in Section II.C. Third, the curvature of a circle (or sphere) undergoes minimal changes during deformation, compared to any other shape because the radius of curvature is constant and equal to the maximum possible value everywhere on the surface. Thus, the change of this radius under strain will be minimal. Preserving a constant curvature of the free surface during deformation causes the surface tension to remain constant during the simulation, which additionally simplifies the surface tension compensation procedure.

\section{The FEM model}

The elastic continuum region was modeled using 8-node hybrid-stress hexahedral finite elements ${ }^{25,26}$ that have a reduced sensitivity to mesh distortion compared to standard displacement-based elements. The constitutive relations were selected as linear elastic to avoid the uncertainty of introducing nonlinear terms in the material constitutive matrix, which are not trivial to estimate from the interatomic potential. The elastic constants in the material constitutive matrix were based on the MD-estimated elastic constants of pure aluminum at $\mathrm{T}=10 \mathrm{~K}$. The values were averaged for uniaxial stresses from 100 to $500 \mathrm{MPa}$ as: $\mathrm{C}_{11}=112.7 \mathrm{GPa}, \mathrm{C}_{12}=59.4 \mathrm{GPa}$ and $\mathrm{C}_{44}=30.6 \mathrm{GPa}$. These values are about 3\% different from the static, zero Kelvin, elastic constants that have been reported for this potential $^{21}$.

The continuum finite element model, as shown in Figure 4, contains an open inner region of diameter $d_{\mathrm{MD}}$, in which to embed the atomistic domain. Along its perimeter, 80 nodes at $z=+h / 2$ and 80 nodes at $z=-h / 2$ were placed to form 160 FEM interface nodes to communicate with the embedded MD domain.

The dimensions of the FEM mesh, $d_{\mathrm{FE}}, d_{\mathrm{MD}}$ and $h$ (see Figure 4) were defined initially through their proportions $d_{\mathrm{FE}}: d_{\mathrm{MD}}: h=20: 1: 1$. Then, the dimensions $d_{\mathrm{MD}}$ and $h$ were rescaled to the equivalent dimensions of the MD system after MD equilibration at zero stress and constant temperature, and after the width of the MD-FEM interface, $l$, and the surface MD region, $s$, had been determined. Finally, the outer dimension of the FEM system $d_{\mathrm{FE}}$ was defined to preserve the ratio $d_{\mathrm{FE}}: d_{\mathrm{MD}}=20: 1$. The outlined procedure allows a single FEM mesh to be applied to various MD systems of different structures and crystallographic orientations through geometric rescaling. This universality of the FEM mesh in the ESCM is a convenient advantage compared to the direct coupling methods ${ }^{1-11}$, in which the FEM mesh has to correlate to the atomic positions of the interface atoms and is specific for each model.

\section{B. Test results and analyses}

Two test cases are considered to interrogate the behavior of the ESCM. First, the stress-strain continuity between MD and FEM domains is validated using the model of an elastically deformed plate with a circular hole. Second, simulating the propagation of an edge crack through the FEM domain into the MD domain is performed to gauge the overall representational capability of the coupled MD-FEM model.

\section{Test for stress-strain continuity over the MD and the FEM regions: elastically deformed plate with a} circular hole

To test the efficiency of the ESCM in transmitting the stress-strain field of a large elastic continuum region to a much smaller MD atomistic region, the classic example of a plate with a circular hole subjected to uniaxial loading was used. In addition to having a simple exact elasticity solution, this model is particularly convenient for two reasons. First, it provides large stress variations (from zero to $3 \sigma_{o}$ ) around the hole, which can be used to test the continuity of the stress field at the MD-FEM interface in the case of large stress gradients. Secondly, keeping the 
peak stress, $3 \sigma_{o}$, well below the theoretical elastic limit of the material (recently estimated for the applied interatomic potential to be between 3 and $5 \mathrm{GPa}^{30}$ ) prevents the occurrence of any plastic mechanisms in the MD domain, which is not addressed in the present study, and a static elastic equilibrium can be achieved everywhere in the system.

To develop the model, a hole of radius $20 \mathrm{~nm}$ is removed from a FE model of dimension $800 \mathrm{~nm}$ by $800 \mathrm{~nm}$ and having properties matching the MD elastic properties for a perfect crystal of aluminum ${ }^{21}$. The elastic anisotropy of the plate follows the crystallographic orientation of the atomistic monocrystalline lattice in the MD domain given in Section III.A.1. The aluminum material from 20 to $40 \mathrm{~nm}$ from the center of the hole is simulated atomistically by a circular MD region with diameter $d_{\mathrm{MD}}=80 \mathrm{~nm}\left(200 a_{\mathrm{o}}\right)$. The MD region is embedded in a continuum elastic square plate of size $d_{\mathrm{FE}}=1.6 \mu \mathrm{m}$ (Figure 4). The square plate is deformed at $0.5 \%$ uniaxial strain along the $x$-direction through fixed displacement boundary conditions imposed on the outer sides of the FEM system $800 \mathrm{~nm}$ away from the hole in the MD domain. At this strain, the far-field stress, estimated as in Figure 5, is $\sigma_{o}=358.5 \mathrm{MPa}$. The stress components in the $z$-direction are kept at zero by controlling the thickness $h$ of the plate using the constant stress Parrinello - Rahmann technique applied along the periodic z-direction in the MD simulation.

Starting from an undeformed MD domain, $\left\{\delta_{I}\right\}=\{0\}$, the coupled MD-FEM iterative simulation is performed, as described in Section II.D, until equilibrium is established between the MD domain and the FEM region $\left(\left\{\delta_{I}\right\}\right.$ and $\left\{R_{I}\right\}$ converge to constant values). The stress field for $\sigma_{x x}, \sigma_{y y}$, and $\sigma_{x y}$ stress components of the coupled MD-FEM system is calculated and compared with a full three-dimensional anisotropic continuum FEM solution, performed using the ABAQUS software package $^{28}$ (Figure 5).

The stress profiles for $\sigma_{x x}$ and $\sigma_{y y}$ along the $x$ - and $y$-axes calculated through the center of the hole are shown in Figures 6(a) and 6(b), respectively. The continuity of the stress profiles is well preserved at the MD-FEM interface apart from fluctuations that result from chaotic atomic thermal vibrations. The magnitude of the thermal fluctuations can be seen in the simulation data of a continuous plate corresponding to the MD-FEM model shown in Figure 5 but without an open hole and subjected to the same far-field strain $\left(\sigma_{x x}\right.$ (plate) and $\sigma_{y y}$ (plate) in Figures 6(a) and 6(b)). In Figures 6(a) and 6(b), the symbols represent the ESCM simulation data, while the full dark lines represent the fully continuum ABAQUS simulation data without surface tension. The stress profiles of the coupled MD-FEM model follow closely the stress profiles of the fully continuum FEM analysis of the same model. The largest discrepancy is observed at the edges of the circular hole. One reason for this discrepancy is the surface tension that exerts a tensile force towards the center of the hole in the MD simulation. Another reason may be because the virial stress gives poor convergence and erroneous estimates near free surfaces $^{29}$.

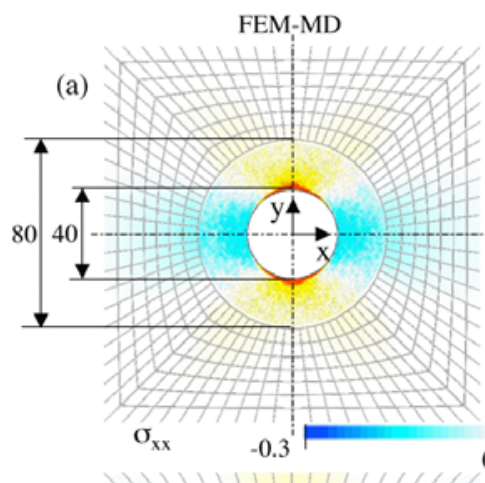

(c)

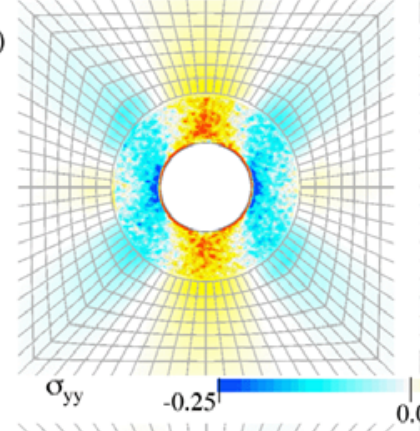

(e)

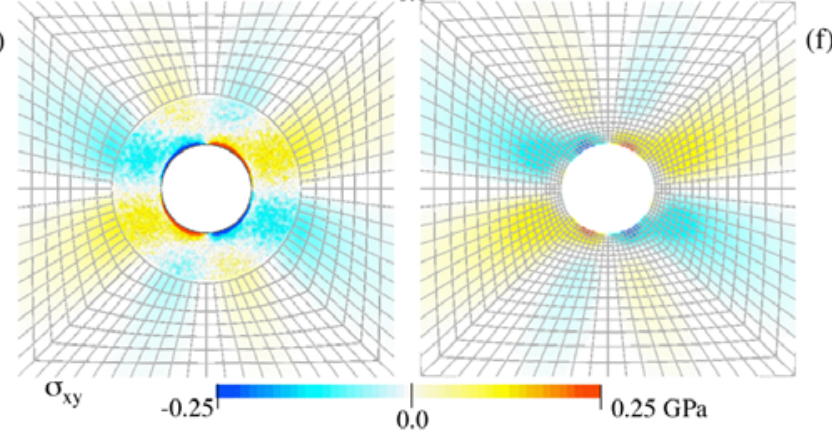

Figure 5. Comparison of stress calculation in MD-FEM and FEM models. 


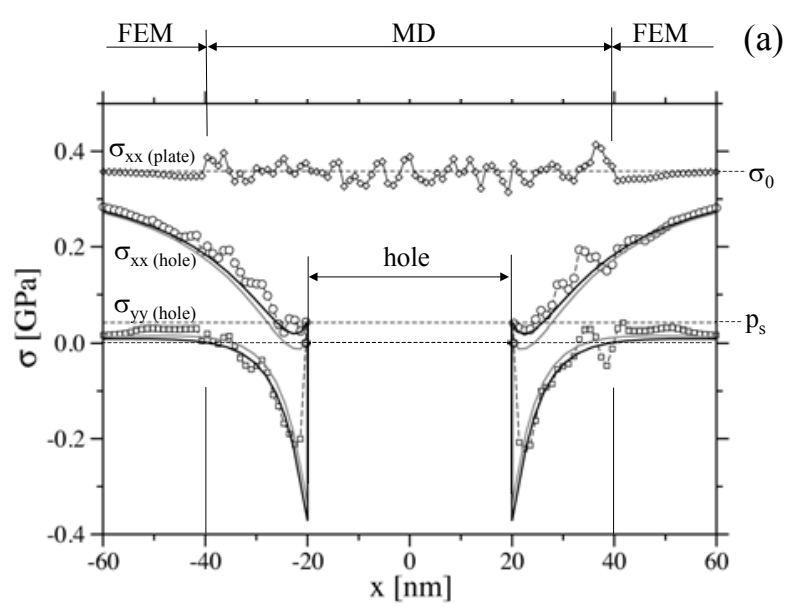

(a) Stresses along a cut in the $x$-direction.

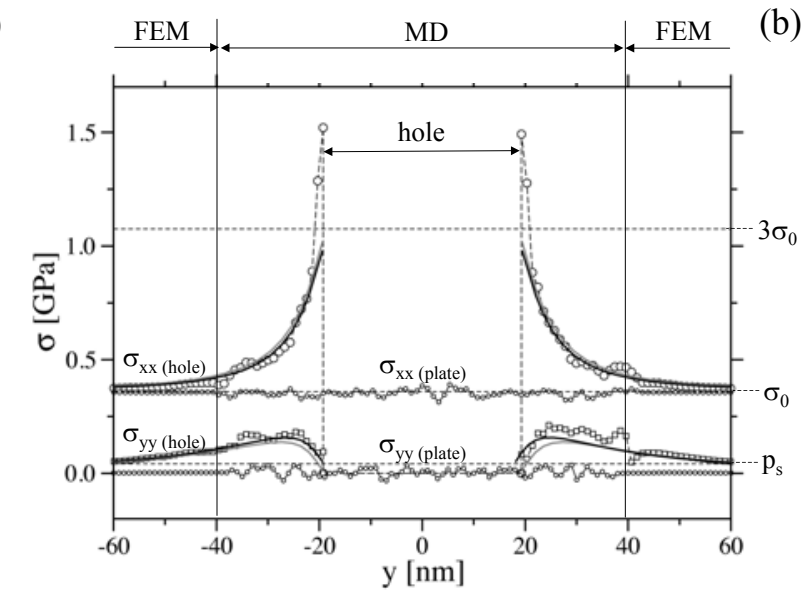

(b) Stresses along a cut in the y-direction.

\section{Figure 6. Comparison of stresses between MD and FEM models along cuts in the $x$ and $y$ directions through the inner open hole.}

In an attempt to account for the surface tension of the hole in the continuum model, an external pressure of $p_{\mathrm{s}}=$ $45 \mathrm{MPa}$ (estimated as $p_{\mathrm{s}}=\gamma / \mathrm{r}$ with $\gamma=0.9 \mathrm{~J} / \mathrm{m}^{2}$, being the energy of the free surface ${ }^{21}$ of the hole, and $r=20 \mathrm{~nm}$, being the hole radius) was applied normal to the surface of the hole in the FEM model. Unlike the continuum simulation without surface tension, the model with surface tension correctly represents the normal component of stress at the hole's surface, which becomes equal to $p_{\mathrm{s}}$ and is plotted using grey lines in Figures 6(a) and 6(b) (following $\sigma_{x x}$ along the $x$-profile in Figure 6(a), and $\sigma_{y y}$ along the $y$-profile in Figure 6(b) when approaching the hole radius at $\pm 20 \mathrm{~nm}$ in both plots). The corresponding MD profiles are in good agreement with the continuum prediction with surface tension. In contrast, the tangential component of the stress ( $\sigma_{y y}$ along the $x$-profile in Figure 6(a), and $\sigma_{x x}$ along the $y$-profile in Figure 6(b)) in the MD system deviates substantially from the continuum prediction when approaching the surface of the hole. As shown in Figure 6(b), the continuum solution closely approaches the theoretical value of $3 \sigma_{o}$ that is calculated for an isotropic material (note that the continuum model in this simulation is anisotropic and some deviation from the isotropic solution is to be expected). As mentioned in the previous paragraph, one reason for this discrepancy may be the virial definition of stress, but more likely it is that the continuum model does not account correctly for the nature of the surface tension, which results from the occurrence of missing bonds between the atoms at the free surface. The effect of missing bonds may result in much stronger local tension between these atoms at the edge than can be considered in the continuum simulation.

\section{Example of an edge crack simulation along a grain boundary in aluminum}

A possible application of the ESCM technique described in this paper is the simulation of atomistic processes where satisfying the correct elastic boundary conditions is of a crucial importance. A typical example is the tip of an edge crack, where the idealized elastic stress field decreases as $1 / \sqrt{r}$ and extends to distances $r$ from the tip far larger than a MD domain can computationally simulate. A coupled FEM-MD model for this example is presented in Figure 7, where the MD domain is used to atomistically simulate the plastic zone at the crack tip, and the FEM region is used to provide the continuum elastic boundary conditions of a far-field tensile strain of $\varepsilon_{y y}=2 \%$ applied along the $y$-direction. To check the validity of the technique at higher temperatures, the simulation was carried out at room temperature $(T=300 \mathrm{~K})$.

The dimensions of the system are: $h=2.9 \mathrm{~nm}, d_{\mathrm{MD}}=45 \mathrm{~nm}$, and $d_{\mathrm{FE}}=900 \mathrm{~nm}$ (see Figure 4). The MD domain represents a bicrystal with a high-angle grain boundary formed between the two crystals, along which the edge crack propagates. In the imposed coordinate system of the model, the orientation of one of the crystals is: $\left(\mathrm{x}:\left[\begin{array}{lll}7 & \overline{7} & \overline{10}\end{array}\right]\right.$; $\mathrm{y}:\left[\begin{array}{lll}5 & \overline{5} & 7\end{array}\right]$; $\left.\mathrm{z:}\left[\begin{array}{lll}1 & 1 & 0\end{array}\right]\right)$, and the orientation of the other crystal is: $\left(\mathrm{x}:\left[\begin{array}{lll}7 & \overline{7} & \overline{10}\end{array}\right] ; \mathrm{y}:\left[\begin{array}{lll}\overline{5} & 5 & \overline{7}\end{array}\right]\right.$; $\left.\mathrm{z:}\left[\begin{array}{lll}1 & 1 & 0\end{array}\right]\right)$. 
In this way, both crystals are mirror images of each other relative to the crystallographic plane $\left\{\begin{array}{lll}5 & 5 & 7\end{array}\right\}$, which becomes the plane of the grain boundary (GB) between them. The GB thus formed is a $<110>\sum 99$ symmetric tilt GB. Crack propagation along this GB has been extensively studied by MD simulations ${ }^{29-31}$ at a cryogenic temperature of $T=100 \mathrm{~K}$. In these studies, it was found that while in one direction the crack becomes blunted by deformation twinning, it propagates in a brittle-like manner in the opposite direction with very little dislocation emission. The latter direction has been chosen as the propagation direction for the edge crack of the simulation in Figure 7. The simulation using the ESCM approach performed at room temperature (Figure 7) showed higher dislocation plasticity than at $T=100 \mathrm{~K}^{30-32}$. The problem remains how to transfer this plasticity to the FEM continuum. Some work in this aspect has been started by Shilkrot et ll. $^{8}$ where they have developed a method to transmit a dislocation from the atomistic region to the continuum region and to follow its propagation using dislocation dynamics simulation in combination with the FEM.

What is essential in the example, provided in Figure 7, is that the developed ESCM approach preserves the continuity of the stress - strain field at the MD-FEM interface even for a dynamic problem such as crack propagation simulated at room temperature. The depicted profile shows a good continuity of stresses across the boundary when the crack speed is slow compared to the elastic response time of the system. In the example shown in Figure 7 , the crack propagation speed was around $100 \mathrm{~m} / \mathrm{s}$ or of the order of $1 / 30$ the speed of sound.

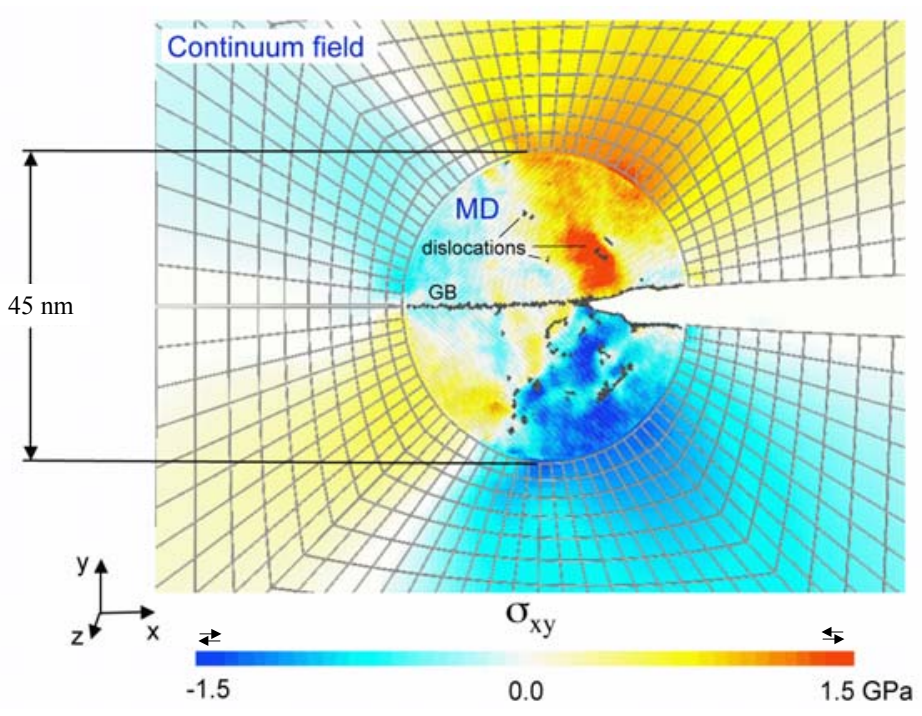

Figure 7. Simulation of an edge crack using ESCM.

\section{Concluding Remarks}

A new statistical approach to coupling MD with FEM simulations denoted embedded statistical coupling method (ESCM) is developed. The approach is based on solving the boundary value problem through an iterative procedure for both MD and FEM systems at their common interface. The two systems are simulated independently and they communicate only through their boundary conditions. The FEM system is loaded along the MD-FEM interface by nodal displacement boundary conditions obtained as statistical averages of the atomic positions in the MD system at the mass centers of associated interface volume cells (IVCs). The MD system is simulated under constant traction boundary conditions obtained from the FEM system as reaction forces to the MD displacements at the interface. This approach allows the continuity at the MD-FEM interface to be achieved at different scales inherent to both systems without the need of redefining continuum quantities at the atomic scale and atomic quantities at the continuum scale, thus avoiding a theoretically controversial concept.

The ESCM shows excellent convergence for systems simulated under static elastic equilibrium, and preserves stress continuity at the MD-FEM interface for systems exhibiting relatively slow dynamics. Additional study needs to be performed to verify if the implementation of a dynamic FEM simulation can improve the dynamics of the system away from equilibrium.

Compared to direct coupling methods ${ }^{1-11}$, ESCM does not represent the MD-FEM interface down to atomistic dimensions but uses statistical mapping of atomistic behavior to continuum FEM representation. ESCM presents a simple and flexible technique in providing elastic boundary conditions for an MD model, thus solving to a large extent the finite-size artifacts inherent in an atomistic simulation. By using statistical averaging to reduce the degrees of freedom when passing information from the atomistic system to the continuum model, the FEM mesh can be independent of the atomic structure of the MD system, and the same FEM mesh can be used with MD models of different size and microstructure without the necessity of remeshing during the simulation. This may prove a 
helpful advantage when plasticity is considered. There is no limitation on the temperature of the atomistic system as long as the thermal corrections to the elastic properties of the continuum system are considered.

From a computational point of view, there are several differences between the ESCM and DC approaches. First, because the FEM mesh is not refined down to the atomic scale at the interface, a significant computational advantage can be obtained when compared to existing DC methods because the size of the finite element model can be decreased by orders of magnitude. Thus, computational resources can be focused on the atomistic calculations yielding the possibility of embedding large MD domains on the order of millions or billions of atoms within the overall material model. In general, an increase in the size of the MD domain associates more atoms with each FEM node in the interface region and improves the continuity at the MD-FEM interface due to improved statistical averages. The integration of detailed atomistic resolution and relatively coarse refinement of the FEM continuum at the interface enables the construction of large models that may benefit engineering applications in the area of nanomechanics such as the study of nano-electromechanical systems (NEMS), integrated circuits, and thin film structural configurations.

Second, because the mesh does not have to correlate with the atomic structure at the interface, it may be used with different MD domains of different atomic microstructure. This independence of the microstructure could be very useful in considering plastic processes at the interface, as no remeshing is needed if atoms experience plastic flow. Also, from a practical standpoint, universal meshes can be prebuilt with a fixed interior region used to embed the MD domain. Thus, various MD systems used to study different atomistic processes within the same overall material model can be repeatedly used without having to construct a new FEM mesh for every different atomic configuration.

Additionally, the method is relatively easy to implement. Any conventional FEM code, including commercial packages, can be used to solve the FEM part of the model. Small modifications to perform the constant traction MD simulation (outlined in Section II.A) are necessary to an otherwise conventional MD code. Thus, the method may be suitable for use in nanoengineering applications where small regions of special interest can be simulated at the atomic level while coupled to a larger material system that can be represented as a continuum.

\section{Acknowledgments}

V. Yamakov is sponsored through cooperative agreement NCC-1-02043 with the National Institute of Aerospace.

\section{Reference}

${ }^{1}$ V Abraham, F.F., Broughton, J., Bernstein, N., and Kaxiras, E., "Spanning the Continuum to Quantum Length Scales in a Dynamics Simulation of Brittle Fracture," Europhys. Lett., 44, 1998, pp. 783-787.

${ }^{2}$ Broughton, J., Abraham, F.F., Bernstein, N., and Kaxiras, E., "Concurrent Coupling of Length Scales: Methodology and Application," Phys. Rev. B, 60, 1999, pp. 2391-2403.

${ }^{3}$ Curtin, W.A. and Miller, R.E., "Atomistic/Continuum Coupling in Computational Materials Science," Modelling Simul. Mater. Sci. Eng., 11, 2003, pp. R33 - R68.

${ }^{4}$ Park, H.S., Liu, W.K., "An Introduction and Tutorial on Multiple-scale Analysis in Solids," Comput. Methods in Appl. Mech. Engrg., 193, 2004, pp. 1733-1772.

${ }^{5} \mathrm{Li}$, X., E, W., "Multiscale Modeling of the Dynamics of Solids at Finite Temperature", J. Mech. Phys. Solids, 53, 2005, pp. 1650-1685.

${ }^{6}$ Miller, R.R. and Tadmor, E.B., "The Quasicontinuum Method: Overview, Applications and Current Directions,” J. Comp. Aided Mat. Desgn, 9, 2002, pp. 203-239.

${ }^{7}$ Xiao, S.P., Belytschko, T., "A Bridging domain method for coupling continua with molecular dynamics," Comput. Methods Appl. Mech. Engrg., 193, 2004, pp. 1645-1669.

${ }^{8}$ Shilkrot, L.E., Miller, R.E., and Curtin, W.A., "Coupled Atomistic and Discrete Dislocation Plasticity," Phys. Rev. Lett., 89, 2002, p. 025501-1.

${ }^{9}$ Gumbsch, P. And Beltz, G.E., "On the Continuum Versus Atomistic Description of Dislocation Nucleation and Cleavage in Nickel," Mod. Sim. Mat. Sci. and Engrg., 3, 1995, pp. 597-613.

${ }^{10}$ Rudd, R.E., Broughton, J.Q., "Coarse-Grained Molecular Dynamics and the Atomic Limit of Finite Elements”, Phys. Rev. B 58, 2005, pp. R5893-R5896.

${ }^{11}$ Rudd, R.E., Broughton, J.Q., "Coarse-grained molecular dynamics: Nonlinear finite elements and finite temperature”, Phys. Rev. B 72, 2005, pp. 144104-1-32.

${ }^{12}$ Cleri, F., "Representation of Mechanical Loads in Molecular Dynamics Simulations," Phys. Rev. B, 65, 2001, 014107-1-6.

${ }^{13}$ O'Rourke, J., Computational Geometry in C, 2nd Edition, Cambridge University Press, 2001. 
${ }^{14}$ Saether, E., Yamakov, V., Glaessgen, E. H., “An Embedded Statistical Method for Coupling Molecular Dynamics and Finite Element Analyses," (in preparation).

${ }^{15}$ Holian, B.L., Ravelo, R., "Fracture Simulations Using Large-Scale Molecular Dynamics,” Phys. Rev. B, 51, 1995, pp. $11275-11288$.

${ }^{16}$ Schäfer, C., Urbassek, H.M., Zhigilei, L.V., Garrison, B.J., "Pressure-Transmitting Boundary Conditions for MolecularDynamics Simulations,” Comp. Mat. Sci., 24, 2002, pp. 421-429.

${ }^{17}$ Moseler, M., Nordiek, J., Haberland, H., "Reduction of the Reflected Pressure Wave in the Molecular-Dynamics Simulation of Energetic Particle-Solid Collisions," Phys. Rev. B., 56, pp. 15439-15445.

${ }^{18}$ Cai, W., Koning, M., Bulatov, V.V., Yip, S., "Minimizing Boundary Reflections in Coupled-Domain Simulations," Phys. Rev. Lett., 85, 2000, pp. 3213-3216.

${ }^{19}$ Park, H.S., Karpov, E.G., Liu, W.K., "Non-Reflecting Boundary Conditions for Atomistic, Continuum and Coupled Atomistic/Continuum Simulations," Int. J. Numer. Meth. Engng., 64, 2005, pp. 237-259.

${ }^{20}$ Cook, R.D., Malkus, D.S. and Plesha, M.E., Concepts and Applications of Finite Element Analysis, Third Edition, John Wiley \& Sons.

${ }^{21}$ Mishin, Y. and Farkas, D., "Interatomic Potentials for Monoatomic Metals from Experimental Data and Ab Initio Calculations," Phys. Rev. B, 59, 1999, pp. 3393 - 3407.

${ }^{22}$ Allen, M.P., Tildesley, D.J., Computer Simulation of Liquids, Oxford University Press, New York, 1987.

${ }^{23}$ Parrinello, M., Rahman, A., "Polymorphic Transitions in Single Crystals; A New Molecular Dynamics Method," J. Appl. Phys., 52, 1981, pp. 7182-7190.

${ }^{24}$ Nose, S., “A Unified Formulation of the Constant Temperature Molecular Dynamics Method,” J. Chem. Phys. 81, 1984, pp. 511-519.

${ }^{25}$ Pian, T.H.H. and Tong, P., "Relations between Incompatible Displacement Model and Hybrid Stress Model," Int. J. Num. Meth. Engrg., 22, 1986, pp.173-181.

${ }^{26}$ Saether, E. "Explicit Determination of Element Stiffness Matrices in the Hybrid Stress Method," Int. J. Num. Meth. Engrg., 38, 1995, pp. 2547-2571.

${ }^{27}$ Cormier, J., Rickman, J. M., Delph, T. J., "Stress calculation in atomistic simulations of perfect and imperfect solids," J. Appl. Phys. 89, 2001, pp. 99-104.

${ }^{28}$ ABAQUS/Standard User's Manual, Hibbitt, Karlsson, and Sorensen, Inc., 2004.

${ }^{29}$ Zimmerman, J.A., Jones, R.E., Klein, P.A., Bammann, D.J., Webb III, E.B., Hoyt, J.J., “Continuum Definitions for Stress in Atomistic Simulation," SAND Report, Sandia National Laboratory, 2002, SAND2002-8608.

${ }^{30}$ Yamakov, V., Saether, E., Phillips, D. R., Glaessgen, E. H., 2005. "Dynamic Instability in Intergranular Fracture,” Phys. Rev. Lett. 95, 2005, p. 015502-1-4.

${ }^{31}$ Yamakov, V., Saether, E., Phillips, D. R., Glaessgen, E. H., "Molecular-Dynamics Simulation-Based Cohesive Zone Representation of Intergranular Fracture Processes in Aluminum,” J. Mech. Phys. Solids, 54, 2006, pp. 1899-1928.

${ }^{32}$ Yamakov, V., Saether, E., Phillips, D. R., Glaessgen, E. H., "Dynamics of Nanoscale Grain-Boundary Decohesion in Aluminum by Molecular-Dynamics Simulation,” J. Mat. Sci., (in press). 